TEPP RAPPORT DE RECHERCHE

\begin{abstract}
A la recherche des incitations perdues : pour une fusion de la prime d'activité, de la CSG, des cotisations sociales et de l'impôt sur le revenu
\end{abstract}

ETIENNE LEHMANN

$\underline{\text { www.tepp.eu }}$

TEPP - Travail, Emploi et Politiques Publiques - FR CNRS 3435 
A la recherche des incitations perdues...

\title{
A la recherche des incitations perdues : pour une fusion de la prime d'activité, de la CSG des cotisations sociales et de l'impôt sur le revenu ${ }^{1}$
}

\author{
Etienne LEHMANN \\ CRED(TEPP), Université Panthéon-Assas Paris II \\ 12 place du Panthéon \\ 75231 Paris Cedex 05 \\ etienne.lehmann@gmail.com
}

La littérature sur la taxation optimale tend à organiser le raisonnement sur la nécessité d'une réforme fiscale autour des seuls arguments d'équité et d'efficacité. Par exemple, pour l'impôt sur le revenu, « un taux marginal élevé fait du mal au niveau où il est imposé et du bien au-dessus » (Piketty 1997, page 164), dans le sens où une hausse du taux marginal de taxation sur un intervalle de revenu distord l'offre de travail sur cet intervalle, mais permet d'augmenter le niveau des impôts au-dessus. Une telle vision peut être enrichie pour tenir compte des décisions de participation (Diamond (1980), Saez (2002)), des décisions de migration (Lehmann, Simula et Trannoy (2014)), de la présence de chômage due à des rigidités de salaires ou des frictions d'appariement (voir la présentation de la littérature par Boadway et Tremblay (2013)), ou de l'interaction de l'imposition directe des revenus avec une taxation indirecte nonuniforme des différents biens et services consommés (Atkinson et Stiglitz (1976)).

Pourtant, on se rend compte qu'en pratique, les agents économiques ne répondent pas forcément au vrai barème d'imposition, mais seulement au barème d'imposition qu'ils ont compris. L'existence d'une telle dissonance a donné naissance à une économie publique comportementale (Chetty (2015), Farhi et Werning (2015)) qui a pour implication que l'on ne saurait résumer le débat sur la fiscalité optimale à la seule question de savoir quels " robinets » ouvrir ou fermer, c'est-à-dire quels taux de taxe sur quelles tranches de revenus augmenter ou diminuer. Le choix des "tuyaux», c'est-à-dire de l'architecture des différents barèmes de prélèvements obligatoires et de prestation compte aussi. Rechercher une meilleure tuyauterie pour notre système de prélèvements obligatoire peut permettre de dégager des pistes de réformes fiscales susceptibles de transcender les habituels clivages partisans et qui pourront par conséquent être mise en œuvre par-delà les alternances électorales.

Cet article se propose de discuter l'opportunité de réformer l'architecture de notre système de prélèvements obligatoires et de politiques de soutien aux bas revenus d'activité. Il milite pour une convergence à terme des différents dispositifs actuellement en vigueur vers un barème unique sur les revenus d'activité. On pense ici à l'Impôt sur le Revenu (IR), y compris sa décote et la Prime Pour l'Emploi (PPE) qu'il comprend, au «volet activité » du Revenu de Solidarité Active (RSA), à la Prime d'Activité (PA) qui se substituera à la PPE et au RSA-activité à partir du 1er janvier 2016, à la Contribution Sociale Généralisée (CSG), à la Contribution au Remboursement de la Dette Sociales (CRDS) et même certaines cotisations sociales. Dans un premier temps, on rappellera un certain nombre de résultats concernant les effets de la méconnaissance du barème de l'Earned Income Tax Credit (dorénavant EITC, la principale politique de soutien aux faibles revenus d'activité qui a inspiré notre PPE) sur le fonctionnement du marché du travail américain. On examinera dans un second temps la superposition des politiques destinées à soutenir les bas revenus d'activité actuellement en vigueur en France. On montrera l'existence d'un certain nombre d'incohérences, qui sont apparues historiquement parce que chaque dispositif a été gouverné de manière isolée. La troisième partie explorera alors les difficultés que créerait la fusion de ces différents dispositifs en un seul et expliquera

\footnotetext{
${ }^{1}$ La rédaction de cet article a bénéficié de conversations avec de nombreux collègues dont Olivier Bargain, Martin Collet, Clément Carbonnier, Laurence Jacquet et Alain Trannoy que je tiens à remercier. Je reste bien entendu le seul responsable des propos tenus dans cet article.
} 
A la recherche des incitations perdues...

comment on pourra y répondre. Elle formulera ainsi deux propositions alternatives pour refondre les différents barèmes d'une manière plus cohérente.

\section{Les effets de la méconnaissance du barème de l'EITC}

L'Earned Income Tax Credit est la principale politique de soutien aux bas revenus d'activité actuellement en vigueur aux Etats Unis. Son barème fédéral dépend de la composition du foyer. Il est beaucoup plus généreux dès qu'il y a des enfants dans le foyer. Il comprend trois zones, une zone d'entrée, un plateau et une zone de sortie (cf. Figure 1). En 2013, le taux de subvention était de $34 \%$ du revenu d'activité avec un enfant et $40 \%$ avec deux enfants. Le taux marginal de taxation en sortie est plus faible puisqu'il était de $15.98 \%$ avec un enfant et $21.06 \%$ avec deux enfants (d'après l'analyse de Hoynes et Pittel (2015) du barème fédéral pour les personnes célibataires au regard de la déclaration d'impôt à l'IRS). Ce barème peut être complété par des aides supplémentaires au niveau des états ou au niveau local.

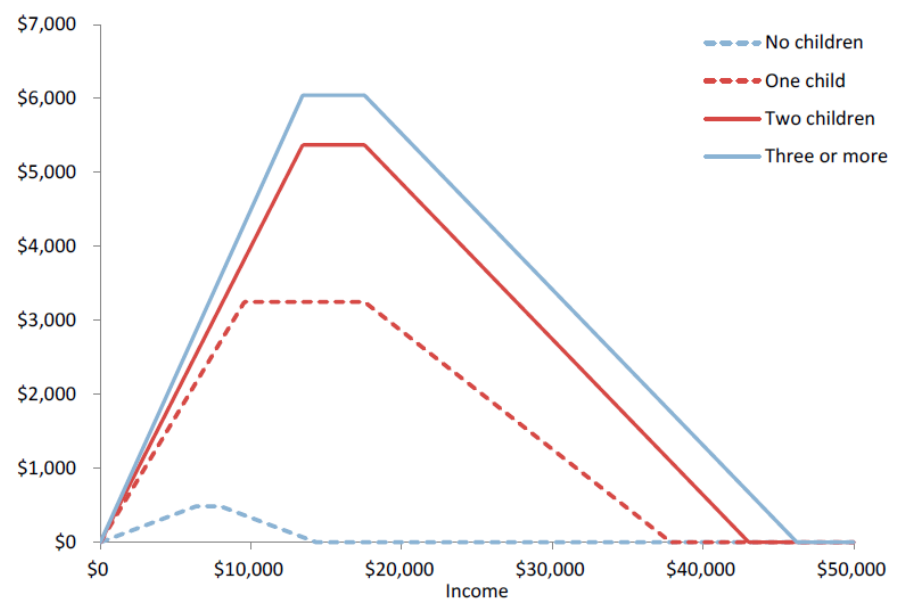

Figure 1 : le barème fédéral de l'EITC en 2013 pour un célibataire selon le nombre d'enfants. Source : Figure 1 de Hoynes et Patel (2015).

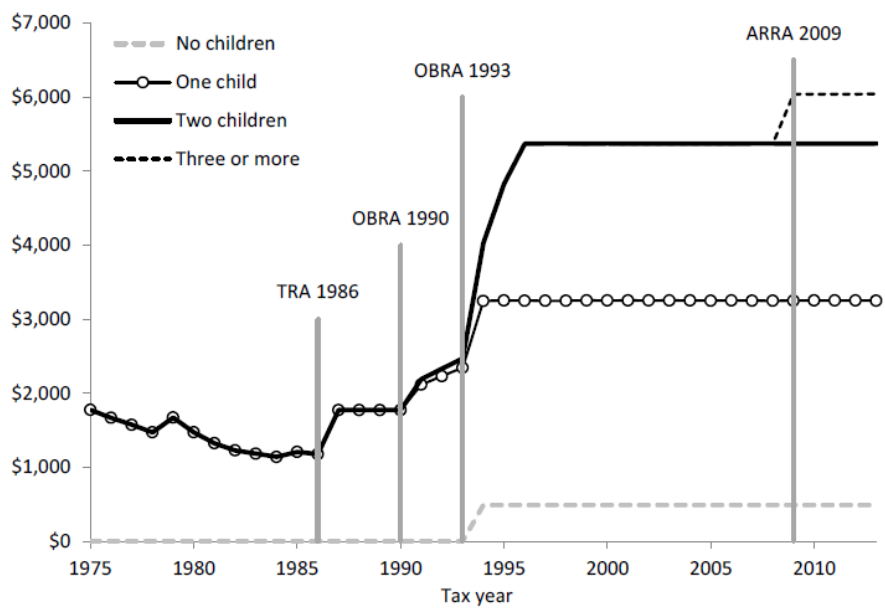

Figure 2 : les évolutions du bénéfice maximal de l’EITC selon l'année fiscale et le nombre d'enfants. Source : Figure 2 de Hoynes et Patel 2015

Le barème fédéral de l'EITC a régulièrement été réformé ainsi que l'illustre la Figure 2. Une réforme particulièrement importante a été mise en œuvre en 1993 suite à l'Omnibus Budget Reconciliation Act (OBRA) de 1993. Cette réforme, qui affecte très différemment les individus selon le nombre d'enfants a fourni un cadre particulièrement approprié pour étudier les effets 
de l'EITC à travers la mise en œuvre d'une identification reposant sur la méthode des différences de différences. Par exemple, Hoynes et Pattel (2015) trouvent qu'une augmentation de $1000 \$$ de l'EITC augmente l'emploi des mères célibataires de 7.3 points et une réduction de la proportion des familles monoparentales sous le seuil de pauvreté de 9.4 points.

Saez (2010) a mis en évidence une autre implication de l'EITC. En effet, les revenus d'activité étant fortement subventionnés dans la phase d'entrée de l'EITC, on s'attend à trouver une surconcentration (ou bunching) de la population avec un revenu d'activité se situant au niveau du seuil séparant la phase d'entrée de l'EITC du plateau de l'EITC. On appellera dorénavant ce niveau le " premier seuil de l'EITC » par opposition au deuxième seuil séparant le plateau de la phase de sortie. Cette surconcentration au niveau du premier seuil correspondrait à la population qui trouverait optimal de travailler un peu plus que le premier si le premier seuil était plus élevé si bien que les revenus supplémentaires resteraient subventionnés, mais qui souhaiterait travailler un peu moins que ce seuil si ce seuil était moins élevé. La figure 3 reporte la distribution des revenus d'activité construite par Saez (2010) pour les familles avec un enfant. $^{2}$ Elle constate effectivement un saut de densité au niveau du premier seuil de l'EITC séparant la phase d'entrée et la phase de plateau. Néanmoins, ce phénomène de surconcentration de la population ne se reproduit pas au seuil séparant le plateau de la phase de sortie. Saez suggère que cette absence s'explique par le fait que la différence entre les taux marginaux entre les deux zones est moins saillante. Enfin et surtout, cette surconcentration n'apparait que pour la population des «travailleurs indépendants » dont au moins une partie des revenus d'activité provient d'une activité à leur compte. Cela suggère que cette population des travailleurs indépendants a beaucoup plus de latitude que la population purement salariée pour ajuster ces revenus d'activité. Cela suggère également que le principal canal de réponse mis en évidence par cette méthode repose sur des réponses en termes de déclarations de revenus et non sur des réponses de l'offre de travail.

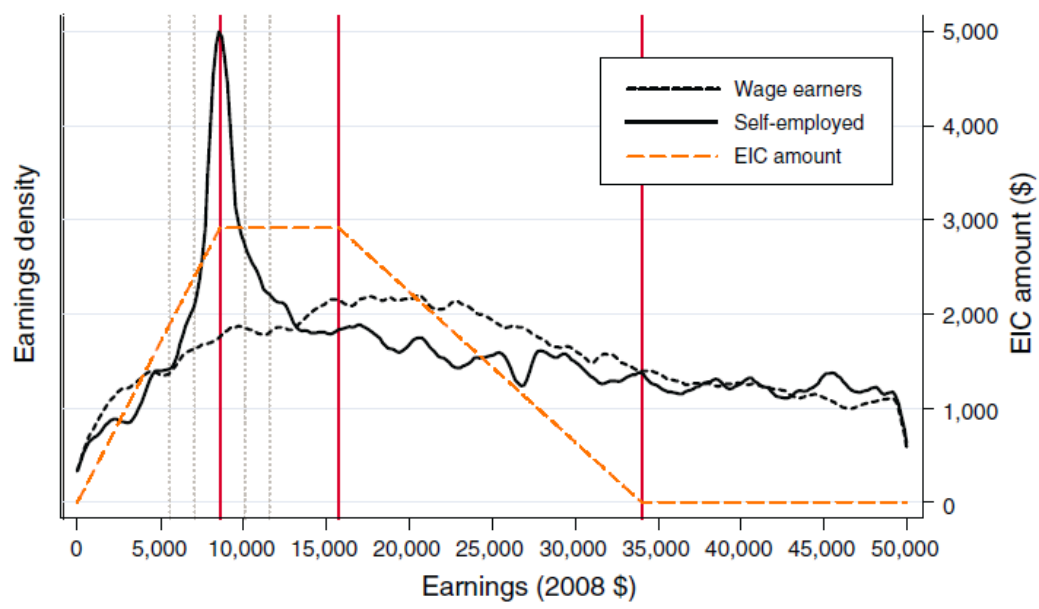

Figure 3 : Distribution des revenus d'activité avec un enfant versus le barème de l'EITC en 2008. Source, Saez (2010), Figure 4 panel A

Chetty, Friedman et Saez (2013) ont alors étudié si ce phénomène de surconcentration de la distribution des revenus d'activité des travailleurs indépendants présentait des variations géographiques et temporelles aux États-Unis. Pour cela, ils ont calculé pour chaque année et chaque zone géographique un indicateur de surconcentration des revenus autour du premier seuil de l'EITC. ${ }^{3}$. Les figures reportent la valeur de cet indicateur, une couleur plus foncée

\footnotetext{
2 Pour être précis, il s'agit d'une estimation des densités par la méthode des noyaux (kernel) incluant toutes les années de 1995 à 2004, une fois inflatés les revenus aux valeurs de 2008. Cette estimation correspond à une version lissée d'histogrammes décrivant la répartition par revenu d'une population.

${ }^{3}$ Les zones géographiques retenues reposent sur les trois premiers chiffres du code postal (ZIP_3), ce qui correspond par exemple à l'ensemble du réseau métropolitain de Boston pour la zone 021. Les auteurs
} 
indiquant un phénomène plus intense de surconcentration. La figure 4 correspond à l'année 1996 et la figure 5 à l'année 2008.

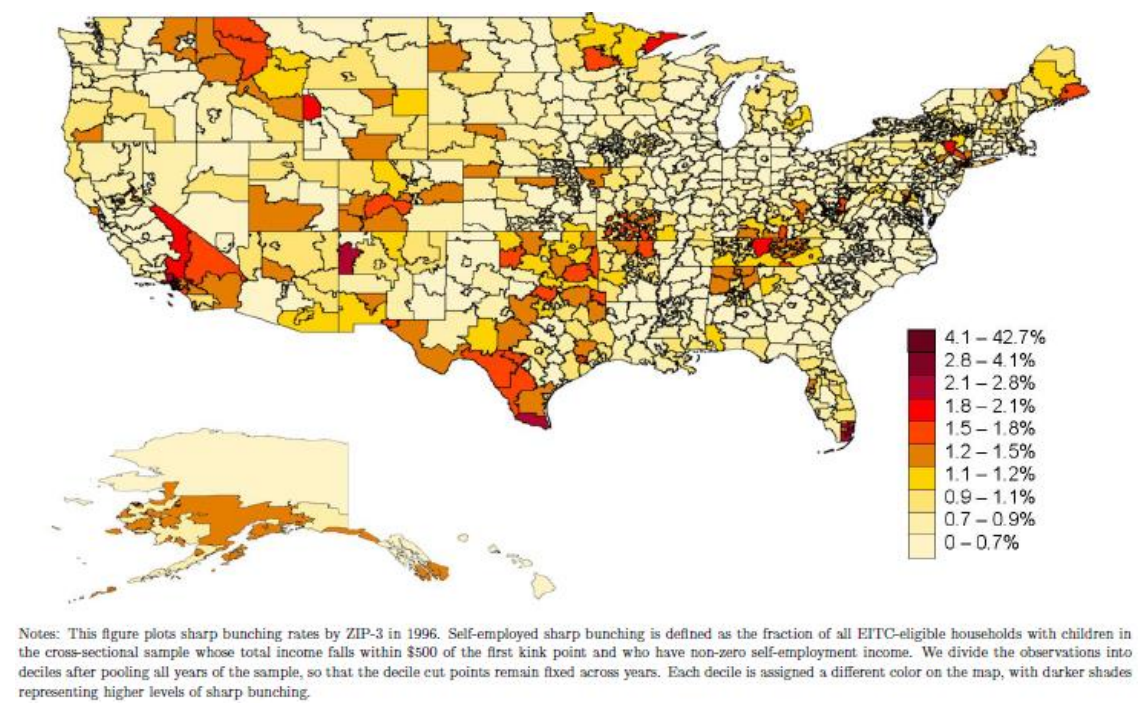

Figure 4 : Intensité de la surconcentration des revenus des indépendants autour du premier seuil de l'EITC en 1996. Source, Chetty et al. (2013), Figure 3 de l'Annexe.

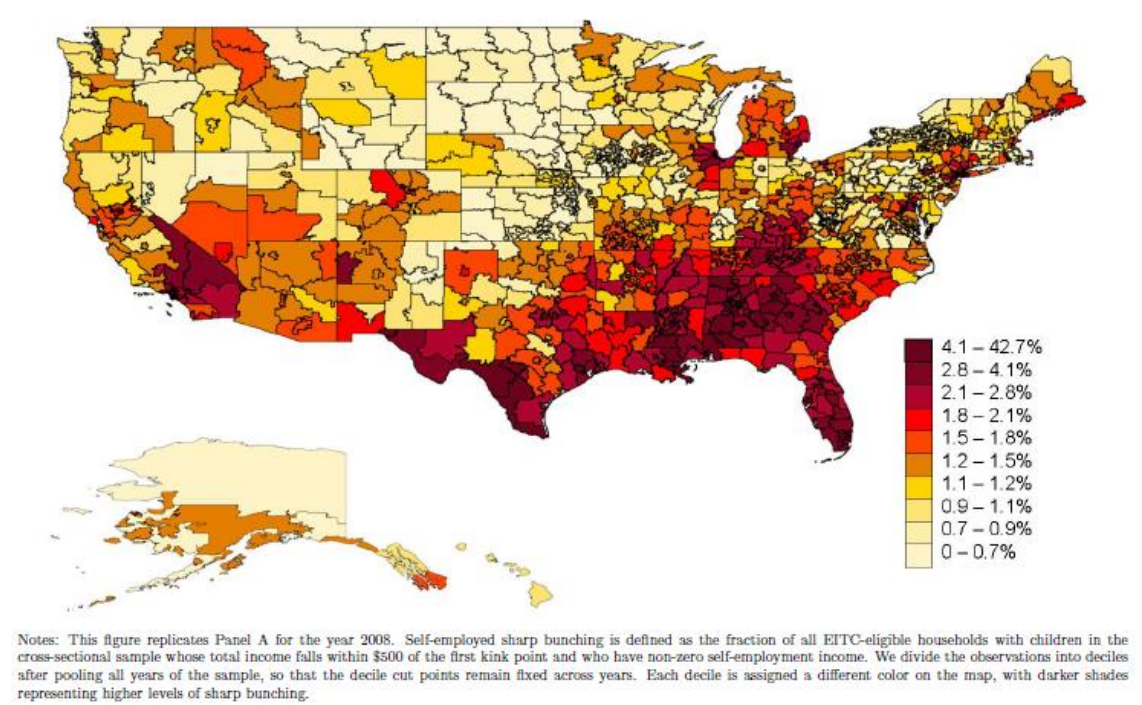

Figure 5 : Intensité de la surconcentration des revenus des indépendants autour du premier seuil de l'EITC en 2008. Source, Chetty et al. (2013), Figure 3 de l'Annexe.

En 1996, le phénomène de surconcentration des revenus des travailleurs indépendants n'apparaissait que dans de très rares zones comme le sud du Texas et les agglomérations Newyorkaise et de Miami. En revanche, douze ans plus tard, le phénomène s'est nettement généralisé. Néanmoins, il subsiste des zones importantes, notamment dans le Nord du « Middle West » où le phénomène reste très faible. Tout se passe comme si à la suite de la réforme OBRA de 1993, les travailleurs avaient progressivement appris qu'ils pouvaient bénéficier d'une subvention intéressante si leurs revenus se situaient exactement au niveau du plateau de l'EITC. Tout se passe également comme si cet apprentissage avait été caractérisé par des effets de voisinage. Chetty et al (2013) montrent ainsi que la distribution des revenus des travailleurs indépendants migrant de zones à forte concentration vers des zones à faibles concentration

obtiennent alors 899 zones géographiques. L'indicateur de surconcentration des revenus retenu est le pourcentage de bénéficiaires de l’EITC reportant des revenus situés à plus ou moins $500 \$$ du premier seuil de l’EITC parmi la population avec au moins un enfant et reportant des revenus d'activité non salarié. 
continuait de rester très concentré, alors que la distribution des revenus des travailleurs indépendants migrant de zones à faible concentration vers des zones à forte concentration devenait au contraire plus concentrée autour du premier seuil.

Chetty et al (2013) ont alors utilisé ces différences dans pour identifier les réponses sur le marché du travail. Leur idée est la suivante. Les zones dans lesquelles la distribution des revenus des travailleurs indépendants ne montre pas de concentration significative autour du premier seuil de l'EITC sont vraisemblablement des zones où les agents ignorent toujours les bénéfices qu'ils peuvent retirer de l'EITC. Elles peuvent alors être utilisées comme approximation de la situation contrefactuelle dans laquelle l'EITC n'existerait pas. Chetty et al. obtiennent alors des élasticités de réponses comportementales tout à fait significatives, notamment le long de la marge intensive. En particulier, pour la population purement salariée, ils obtiennent des estimations statistiquement significatives de l'élasticité intensive autour de valeurs comprises entre 0.2 et 0.5 . (cf. Table 3 page 2715 de Chetty et al (2013)).

On le voit à travers cet article, une réforme fiscale peut mettre beaucoup de temps à produire ses effets parce que les agents mettent du temps à comprendre quelles incitations sont contenues dans le vrai barème auquel ils font face. Nous allons donc à présent examiner les différents dispositifs ayant un impact sur le bas des revenus d'activité actuellement en vigueur en France et discuter de leur cohérence.

\section{Une superposition de dispositifs souvent sources d'incohérences}

L'impôt sur le revenu des personnes physiques (IR) est probablement le prélèvement obligatoire qui occupe le plus d'espace dans le débat public en France. Pourtant, ses recettes ne se montent qu'à $3 \%$ du PIB là où le seul impôt fédéral sur le revenu représente $6,7 \%$ aux Etats Unis en 2011. ${ }^{4}$ Un tour d'horizon international confirme cette idée que l'IR français est l'un des moins rentables dans les pays de l'OCDE. On peut expliquer ce phénomène par le fait qu'il n'est payé grosso modo que par la moitié des contribuables. La conséquence est qu'il est très concentré, puisque par exemple le décile supérieur de revenu imposable contribue à $70 \%$ pour les recettes totales de l'IR.

Les défauts de l'IR sont bien connus (voir par exemple Landais et al (2011) ou CPO (2015)). Payé au niveau du foyer fiscal et non au niveau des individus, il est l'un des derniers impôts sur le revenu parmi les pays de l'OCDE à ne pas être prélevé à la source, en attendant la réforme de 2017. Comme ce sont les contribuables qui sont donc directement redevables à l'IR, il concentre sur lui une bonne partie des discours les plus clientélistes en matière de prélèvements obligatoires. Il n'est donc pas très surprenant de constater qu'il comprend 190 " dépenses fiscales » (le terme officiel pour les niches fiscales) pour un montant estimé à 34 milliards par an (Guillaume (2011)). Si certaines de ces niches peuvent avoir une justification économique à l'instar de la prime pour l'emploi (PPE) qui est aussi considérée comme une dépense fiscale, on peut douter de la pertinence d'un grand nombre d'entre elles. ${ }^{5}$

\footnotetext{
${ }_{4}^{4}$ D’après la table 11in05tr sur le site de l'IRS https://www.irs.gov/pub/irs-soi/11in05tr.xls, les recettes totales de l'impôt sur le revenu se montaient en 2011 à 1 042,571 milliards de dollars alors que le PIB cette année là se montait à 15517,9 milliards de dollars d'après le site du BEA http://www.bea.gov/national/xls/gdplev.xls A cet impôt fédéral s'ajoute les impôts sur le revenu des états.

5 Dans la liste dressée par le rapport Guillaume, on apprend ainsi l'existence de l'« exonération des sommes perçues dans le cadre de l'attribution du prix Nobel ou de récompenses internationales de niveau équivalent au prix Nobel dans les domaines littéraires, artistiques ou scientifique » dont on regrettera que le coût n'ait put être chiffré, sans doute à cause du nombre trop réduit de bénéficiaires.
} 
La CSG est un impôt ${ }^{6}$ affecté au financement de la sécurité sociale qui a été créé en 1991. Aujourd'hui, la CSG sur les revenus d'activité était proportionnelle à un taux de $7.5 \%$ tandis que la CSG sur les revenus du patrimoine et sur les produits de placement était de $8.2 \%$. La CSG sur les revenus de remplacement est progressive avec l'existence d'une exonération de CSG pour les retraités et les chômeurs les plus modestes et d'un taux réduit de 3.8\% pour les retraités et les chômeurs ayant un revenu intermédiaire, le taux normal de CSG sur les revenus de remplacement étant de $6.2 \%$. La CSG est prélevée à la source et de manière contemporaine. Elle est enfin beaucoup moins sujette que l'IR à la multiplication des niches. Sans doute est-ce dû à l'illusion qu'ont les contribuables de ne pas la payer puisqu'elle est prélevée directement sur leur fiche de paie. Aujourd'hui, la recette de la CSG représente plus de 4\% du PIB dépassant ainsi le rendement de l'IR. La Contribution au Remboursement de la Dette Sociale (CRDS) fonctionne selon des principes très similaires avec un taux unique de $0.5 \%$. On assimilera dans la suite de cet article la CRDS à la CSG.

On peut considérer que la CSG constitue un deuxième impôt sur le revenu non officiel.7 Avec cette interprétation, on peut alors être surpris par les argumentaires selon lesquels il serait incivique que seule la moitié des contribuables paye l'impôt sur le revenu. Cette critique resurgit très fréquemment, à l'instar de l'amendement déposé en 2015 par le député socialiste Razzy Hammadi visant à établir un « impôt sur le revenu minimum obligatoire ». C'est oublier que tous les revenus d'activité sont assujettis à la CSG, à la CRDS et aux cotisations sociales (et aussi que tous les consommateurs payent de la TVA). On le voit, le débat fiscal gagnerait en transparence démocratique et éviterait les représentations faussées s'il n'existait qu'un seul impôt.

La focalisation du débat fiscal sur le seul IR a également pour conséquence que la part des foyers qui sont imposables à l'IR est devenu un thermomètre de la pression fiscale sur les classes moyennes et populaires. Or, à la suite du gel des barèmes décidé en 2011, ce nombre s'est accru de manière significative. C'est sans doute pour contrer la critique selon laquelle il « matraquerait » fiscalement les classes moyennes et populaires que le gouvernement Valls a fait voter dans le projet de loi de finances pour l'année 2015 la suppression de la première tranche d'IR à 5\% ainsi que la modification des paramètres de la décote à l'IR. La décote à l'IR est un mécanisme obscur dont le fonctionnement est illustré dans la figure 6. Elle consiste à retarder l'entrée dans le barème en créant officieusement une tranche supplémentaire à un taux supérieur au premier taux apparaissant dans le barème officiel de l'IR. Très concrètement, pour les revenus de 2015 , le barème officiel annonce une première tranche à $14 \%$ pour les revenus entre $9690 €$ et $26764 €$. Mais en tenant compte de la décote, le vrai barème comprend, pour les célibataires, une première tranche à $28 \%$ entre $13744 €$ et $17797 €$ et une deuxième tranche à $14 \%$ entre $17797 €$ et $26764 €^{8} .^{8}$ Alain Trannoy (2015) explique ainsi « de la même

\footnotetext{
${ }^{6}$ Du moins pour le conseil d'état et le conseil constitutionnel, même si la Cour de Justice Européenne le perçoit comme une cotisation sociale, ce qui implique que les contribuables frontaliers résidant en France mais immatriculés à la sécurité sociale d'un autre pays membre de l'UE ne sont pas redevables de la CSG

${ }^{7}$ Le rapport du CPO (2015) sur l'articulation de l'IR et de la CSG relate dans ses pages 27 à 28 puis 127 à 128 un certain nombre d'anecdotes. D’après le compte rendu intégral de la séance de l'Assemblée nationale du 15 novembre 1990, le député François d'Aubert a déclaré « Il serait plus honnête de dire qu'il faut réformer l'impôt sur le revenu en élargissant son assiette que de le faire ainsi de façon clandestine, en douce, par le biais de la CSG ». Christian Poncelet a par ailleurs qualifié la CSG « d'impôt sur le revenu bis » d'après le compte rendu intégral de la séance du Sénat du 9 juin 1993. On apprend également qu'à la fin de l'année 1993, le ministre du Budget de l'époque, Nicolas Sarkozy, indiquait dans la lettre de mission adressée à la nouvelle commission chargée d'étudier les prélèvements fiscaux et sociaux pesant sur les ménages : «vous proposerez les évolutions nécessaires pour simplifier, tant en matière d'assiette que de recouvrement, l'impôt sur le revenu et assurer sa cohérence avec la contribution sociale généralisée et les contributions sociales ».

${ }^{8}$ Sur le site du bulletin officiel des finances publiques, la décote est expliquée comme « Le montant de la décote est égal à la différence entre le plafond applicable en fonction de la situation familiale du contribuable, [...] (1 $135 €$ pour les contribuables célibataires, divorcés ou veufs et $1870 €$ pour les
} 
manière qu'un chasse-neige crée des congères en chassant la neige sur les bas-côtés, la décote agit comme un chasse-contribuable » et crée une véritable congère fiscale en doublant le taux marginal officiel entre $13744 €$ et $17797 €$. On le voit, la décote devient un élément essentiel du barème fiscal pesant sur les revenus d'activité.

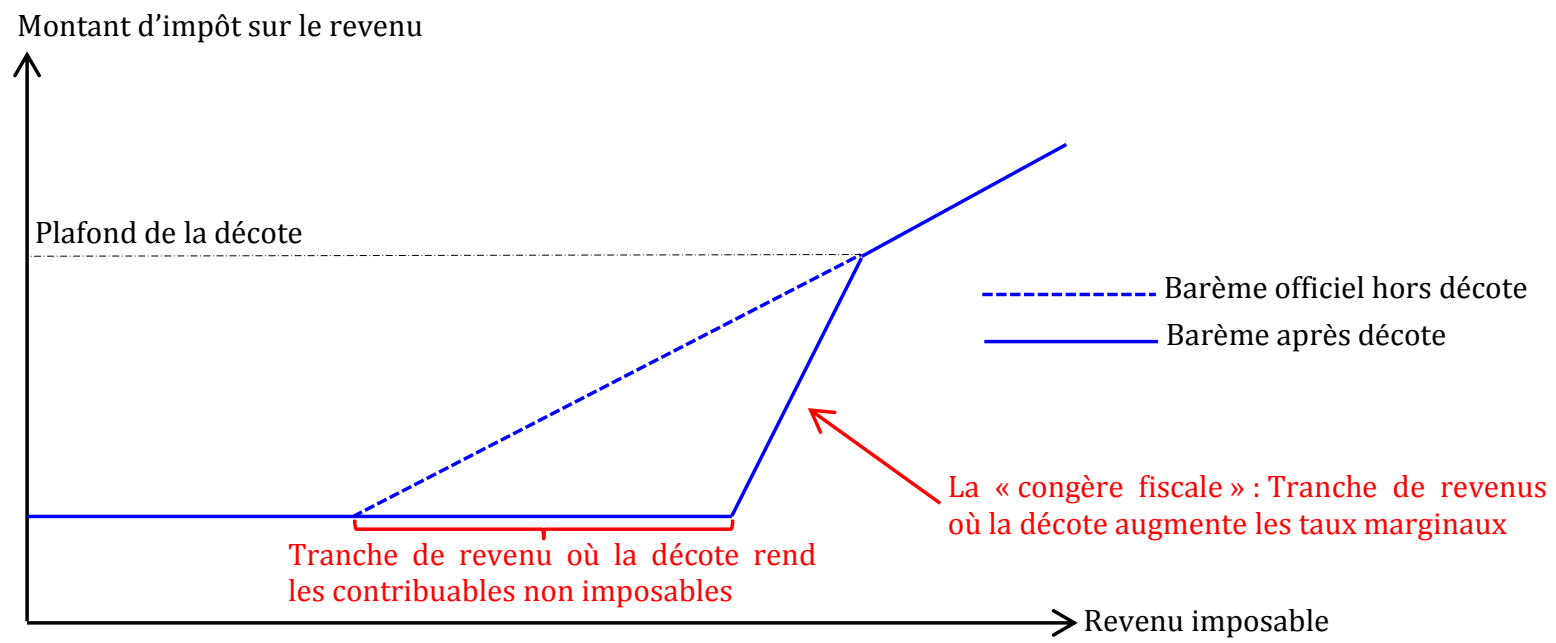

Figure 6 : le principe de la décote

L'impôt sur le revenu comprend enfin dans son barème la prime pour l'emploi (PPE) qui est la version française de l'EITC américain. La prime pour l'emploi est destinée à inciter au retour ou au maintien dans l'emploi. Son barème en 2015 pour les revenus de $2014^{9}$ dépend des revenus de chaque membre du foyer. Il comprend une phase d'entrée à $7.7 \%$ pour les revenus inférieurs à $12475 €$, et une phase de sortie où la prime diminue au taux marginal de 19,3\% pour les revenus compris entre $12475 €$ et $17451 €$. Le seuil de $12745 €$ est réajusté chaque année pour correspondre au revenu imposable d'une personne travaillant à temps plein toute l'année et rémunérée au SMIC, le seuil de $17451 €$ correspondant à 1.4 fois le SMIC. Néanmoins, pour bénéficier de la PPE, les revenus de l'ensemble du foyer fiscal doivent être inférieurs à un seuil qui dépend de la composition du foyer fiscal. Le calcul de la PPE est alors modifié pour tenir compte de la composition du foyer fiscal. La PPE est enfin calculé sur la base d'une activité à temps plein rémunérée tout au long de l'année. Dans le cas d'une activité à temps partiel ou rémunérée une partie seulement de l'année, le barème de la PPE s'applique sur le montant des revenus en équivalent temps plein et le montant de la PPE est ensuite divisé par le rapport entre le nombre d'heures effectivement rémunérées dans l'année et le nombre d'heures rémunérées par un emploi à temps plein tout au long de l'année, c'est-à-dire 1820 heures pour les salariés.

On le voit, le barème de la PPE est complexe car il mêle à la fois des considérations sur l'activité de chaque membre du foyer fiscal et sur la situation de l'ensemble du foyer fiscal. En lisant les textes décrivant le calcul de la PPE, on se dit qu'il aurait été beaucoup plus simple et lisible de se contenter d'une baisse de cotisations sociales salariés sur les bas salaires, à l'instar des allègements de cotisations sociales patronales bas salaires dits « Fillon ». C'était en réalité le

contribuables soumis à imposition commune à compter de l'imposition des revenus de 2014) et l'impôt brut résultant du barème.

Par suite, les contribuables dont l'impôt brut est égal ou inférieur à la moitié du plafond de la décote voient leur impôt brut ramené à $0 €$. Lorsque l'impôt brut est supérieur à la moitié de ce plafond, la réduction d'impôt procurée par la décote diminue au fur et à mesure que le montant de l'impôt brut se rapproche du plafond », http://bofip.impots.gouv.fr/bofip/2495-PGP . 9

http://www.legifrance.gouv.fr/affichCodeArticle.do?idArticle=LEGIARTI000029042107\&cidTexte=LEGIT EXT000006069577\&dateTexte $=20140530 \&$ oldAction $=$ rechCodeArticle\&fastReqId $=675869077 \&$ nbesul $\underline{\operatorname{tRech}=1}$ 
projet initial du gouvernement Jospin qui voulait instaurer une ristourne progressive de CSG sur les salaires inférieurs à 1.4 SMIC. Mais un tel projet a été censuré par le Conseil Constitutionnel au motif notamment qu'elle ne tenait pas compte des revenus des autres membres du foyer fiscal. ${ }^{10}$ C'est pour contourner la censure par le Conseil constitutionnel du projet initial de ristourne dégressive de CSG que le barème de la PPE a introduit des éléments touchant aux revenus du foyer. ${ }^{11}$ Collet (2014) relate comment le Conseil constitutionnel, par sa jurisprudence a multiplié un certain nombre de principes qui s'impose aujourd'hui au législateur.

On le voit, la PPE est un dispositif complexe. Elle est maximale au niveau du SMIC, mais elle procure un avantage beaucoup plus faible que l'EITC américain. Au premier seuil de l'EITC, celui-ci augmente les revenus de 34\% (avec un enfant). En France, la subvention n'est que de 7.7\%. Elle est même légèrement inférieure à la CSG et la CRDS qu'un Smicard doit acquitter. De plus, elle se matérialise par une baisse de l'impôt sur le revenu ou par un crédit d'impôt. Avant le passage au prélèvement à la source de l'IR, le bénéfice de la PPE est donc versé avec un an de retard. Ainsi, comme l'ont notamment souligné Landais et al. (2011), tout se passe comme si chaque mois de l'année $t$, on prélevait $8 \%$ sur les salaires d'un smicard au titre de la CSG et de la CRDS, pour lui rembourser quasiment intégralement à la fin de l'année $t+1$. Difficile d'imaginer compte tenu de ce décalage temporel de versement que la PPE puisse avoir des effets par exemple pour inciter des personnes qui ne se verraient proposer que des contrats courts (disons un mois ou moins) rémunérés au SMIC d'accepter un plus grand nombre de propositions dans l'année. Le décalage temporel conjugué à des contraintes de liquidité qui frappe trop souvent ces populations font vraisemblablement disparaitre tout effet bénéfique. De tels doutes sont renforcés justement par les résultats de Chetty et al (2013).

Le revenu de solidarité active (RSA) a été mis en place en 2009 en remplacement du revenu minimum d'insertion (RMI). Selon Bargain (2014), on peut voir l'ensemble du dispositif du RSA comme une aide différentielle au revenu qui taxerait à $38 \%$ les revenus des ménages là où le RMI les taxait à $100 \%$ jusqu'à atteindre un « revenu minimum garantit » dont le montant dépend également de la composition du ménage. Le RSA est versé mensuellement. Le volet « activité » du RSA, c'est-à-dire la partie du dispositif qui sert à garantir que $62 \%$ des revenus d'activités des bénéficiaires du RSA leur soit maintenue, a donc l'avantage sur la PPE de réduire le décalage dans le temps entre la perception du revenu d'activité et la perception de l'aide. Mais son bénéfice n'est pas automatique puisqu'il faut faire une demande auprès de la Caisse d'Allocation Familiale. Le bénéfice du RSA est alors examiné sur la base d'une déclaration de revenu trimestrielle. Bargain (2014) indique alors que le taux de non-recours au RSA activité s'élèverait à $68 \%$. Un dispositif incitant à la reprise d'activité qui ne serait versé qu'à un tiers des bénéficiaires potentiels n'est sans doute pas très efficace en termes d'incitations au retour à l'emploi.

La mise en place du RSA s'est opérée en remplacement du Revenu Minimum d'Insertion (RMI), mais pas en remplacement de la PPE. Les barèmes de la PPE et du RSA activité ne sont pas

\footnotetext{
10 Le point 9 de la décision $n^{\circ} 2000-437$ du 19 décembre 2000 du conseil constitutionnel indique notamment que «Considérant que, s'il est loisible au législateur de modifier l'assiette de la contribution sociale généralisée afin d'alléger la charge pesant sur les contribuables les plus modestes, c'est à la condition de ne pas provoquer de rupture caractérisée de l'égalité entre ces contribuables; que la disposition contestée ne tient compte ni des revenus du contribuable autres que ceux tirés d'une activité, ni des revenus des autres membres du foyer, ni des personnes à charge au sein de celui-ci ; que le choix ainsi effectué par le législateur de ne pas prendre en considération l'ensemble des facultés contributives crée, entre les contribuables concernés, une disparité manifeste contraire à l'article 13 de la Déclaration de $1789 »$.

${ }^{11} \mathrm{Si}$ on considère que l'incidence économique des allègements de cotisations patronales sur les bas salaires est d'augmenter les salaires, le Conseil constitutionnel aurait dû répliquer le raisonnement qui l'a conduit à censurer la ristourne dégressive de CSG pour censurer les allègements de cotisations patronales bas salaires, ce qu'il n'a heureusement pas fait.
} 
identiques mais procurent des bénéfices similaires au niveau d'un SMIC à temps plein. Le RSA activité est plus généreux pour les emplois au SMIC à temps partiel et moins généreux que la PPE au-delà du SMIC. En pratique, pour les bénéficiaires du RSA activité, le montant du RSA vient en déduction de la PPE. La figure 7

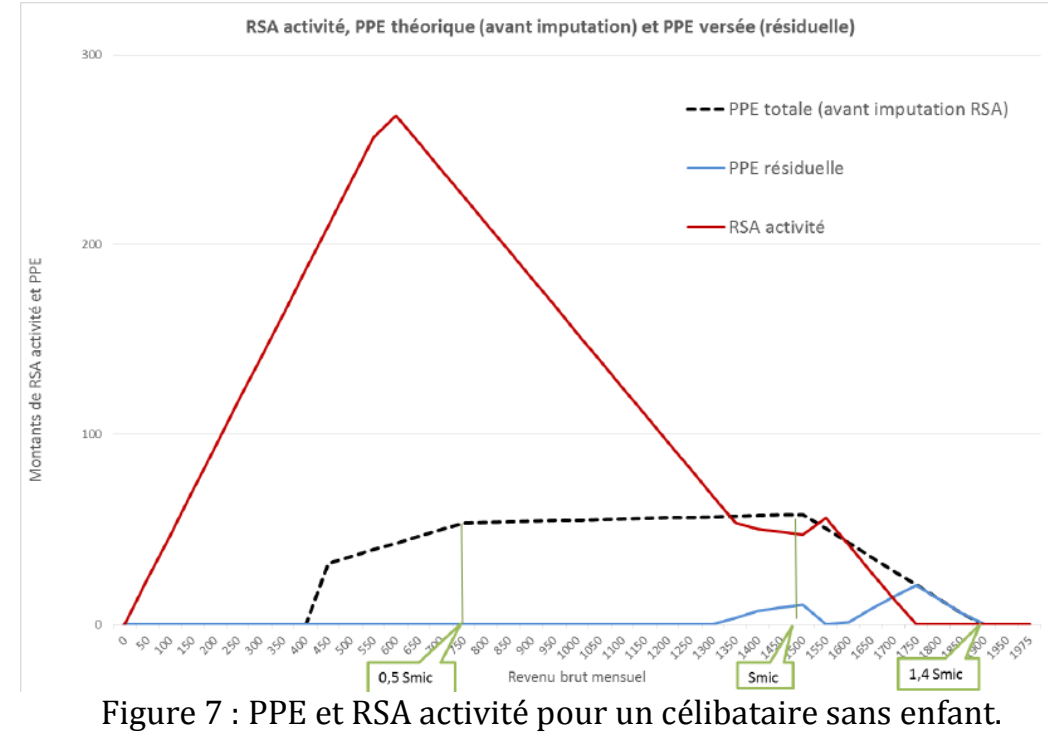

Source Bargain (2014, graphique 10)

C'est du moins la situation qui prévalait avant la mise en place de la prime d'activité (PA) au $1^{\text {er }}$ janvier 2016 qui va fusionner RSA activité et PPE en un seul dispositif. On peut malheureusement s'attendre à ce que la PA hérite des défauts du RSA. Comme son attribution ne sera pas automatique mais se fera comme pour le RSA sur la base d'une déclaration à la CAF, un fort taux de non recours est donc à craindre. Il ne faut pas perdre de vue que ces taux de non recours aussi élevés, mêmes si il révèle un manque de transparence sur les possibles incitations à la reprise d'activité, constituent également une source d'économie non négligeable pour les finances publiques.

La politique familiale constitue une autre source importante de revenus pour les familles modestes. La politique familiale ne s'adresse pas qu'aux familles modestes mais à l'ensemble des familles. Elle s'exerce pourtant à l'aide d'instruments différents. Les familles modestes sont concernées en tout premier lieu par l'indexation du RSA et des allocations logement sur le nombre d'enfants alors que les ménages aisés sont davantage concernés par le mécanisme du quotient familial. Ce dernier fonctionne comme suit. Au lieu d'appliquer le barème de l'impôt sur le revenu à l'ensemble des revenus du foyer, on ne l'applique qu'au quotient familial qui est égal au rapport entre le revenu imposable du foyer sur le nombre de parts. Un couple sans enfants bénéficie de deux parts, un couple avec un enfant de deux parts et demi, etc. L'impôt dû par le foyer est alors égal au produit de l'application du barème à ce quotient familial par le nombre de parts du foyer. Le quotient familial agit de ce point de vue comme une aide aux familles dont le montant serait proportionnel (à tout le moins croissant) avec le revenu des familles. C'est pourquoi le bénéfice du quotient familial (c'est à dire pour un couple avec enfants, la différence entre l'impôt dû et l'impôt qu'il devrait s'il n'avait que deux parts) est plafonné. Le plafond du quotient familial a été fortement réduit pour atteindre $1508 €$ en 2015 pour chaque demi-part d'une personne à charge. A contrario les allocations familiales sont forfaitaires en apportant le même montant aux familles quels que soient leur revenus.

Le caractère multiforme de la politique familiale implique que l'on a du mal à avoir une vision d'ensemble. On remarque alors que la combinaison des allocations familiales, de l'IR (y compris la PPE, sa décote et son quotient familial), de l'allocation de rentrée scolaire, de la taxe d'habitation, du complément familial et de l'ensemble allocations logements et RSA aboutit à ce 
que d'avoir un enfant procure un revenu disponible supplémentaire quasiment insensible avec le niveau de revenu imposable. C'est ce que montre par exemple la Figure 8 ci-dessous. Un tel résultat a notamment été obtenu grâce à l'abaissement du plafonnement du quotient familial. On voit alors se dessiner une piste de réforme de simplification qui consisterait à supprimer l'attribution de part supplémentaire pour chaque enfant d'un couple ainsi que l'indexation des différentes prestations non familiales pour basculer l'intégralité de la politique familiale sur les seules allocations familiales sans condition de ressources. Une telle réforme permettrait non seulement de gagner en lisibilité et donc en transparence du débat démocratique, mais elle permettrait également d'éviter que la politique familiale ne vienne contaminer la politique d'incitation à la reprise d'activité en renforçant d'éventuels trappes à bas salaires.

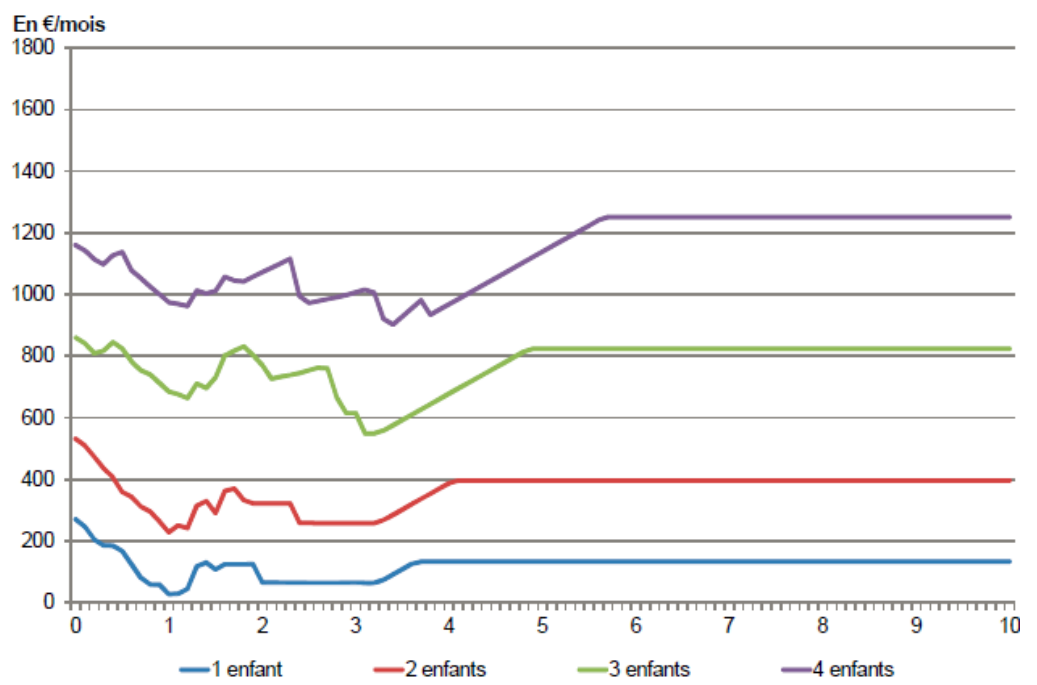

Figure 8 : Supplément de transfert lié au nombre d'enfants pour un couple biactif en fonction du revenu du 1 er conjoint. Source, Graphique 1 de Boisnault et Fichen (2015)

Dans ce panorama des politiques de soutien aux bas revenus, on peut être surpris par le rôle joué par les allocations logement (AL). Les allocations logements sont versées à des ménages locataires sous condition de ressources. Leur montant dépend à la fois des caractéristiques du logement, de la composition du ménage et des revenus du ménage. Bargain (2014) note que les allocations logement peuvent représenter jusqu'à un $1 / 3$ des ressources des personnes privées de revenus. Un peu comme pour la politique familiale, la politique d'aide au logement vient donc brouiller les incitations à la reprise d'activité en voulant réserver le bénéfice des AL aux ménages les plus défavorisés. Puisque l'objectif est ici redistributif, on peut donc se demander pourquoi les AL doivent dépendre du loyer plutôt que de déconnecter le montant des AL des loyers payés et de les fondre dans le barème d'ensemble relatif aux revenus d'activité. En pratique 86\% des locataires du parc locatif privé ont un loyer qui dépasse le loyer plafond (Bargain (2014)). Le montant de leur AL est alors indépendant du montant de leur loyer. Cela permet de limiter les effets des AL sur les loyers qui ont notamment été documentés en France par Fack (2006): 78\% des bénéfices des allocations logements aboutirait à une augmentation des loyers si bien que seule $22 \%$ bénéficierait effectivement aux locataires Néanmoins, une telle réforme nécessite probablement d'être mise en œuvre de manière graduelle compte tenu justement de l'impact inflationniste estimé des allocations logement sur les loyers. L'idée est de procéder par petits pas pour ne déclencher l'étape suivante du basculement des ressources des AL vers des transferts non conditionnés aux les dépenses de logement qu'une fois effectivement constaté une baisse des loyers.

Pour conclure ce tour d'horizon des différents dispositifs, il peut être intéressant de simuler l'ensemble du dispositif. C'est ce que propose la Figure 9 dans le cadre très simple d'un hypothétique célibataire sans enfant (il ne touche donc pas d'allocation familial), sans allocation 
logement (par exemple parce que propriétaire de son logement par héritage), ne recevant que des revenus d'activité et qui ne recours pas au RSA activité. Il s'agit donc d'un cas type très hypothétique et particulièrement simple. Notre cas-type n'est donc concerné que par l'impôt sur le revenu, la décote et la prime pour l'emploi. ${ }^{12}$ La courbe la plus épaisse correspond à la combinaison de ces trois barèmes. On reporte sur l'échelle de droite le taux moyen et marginal associé à cette combinaison.

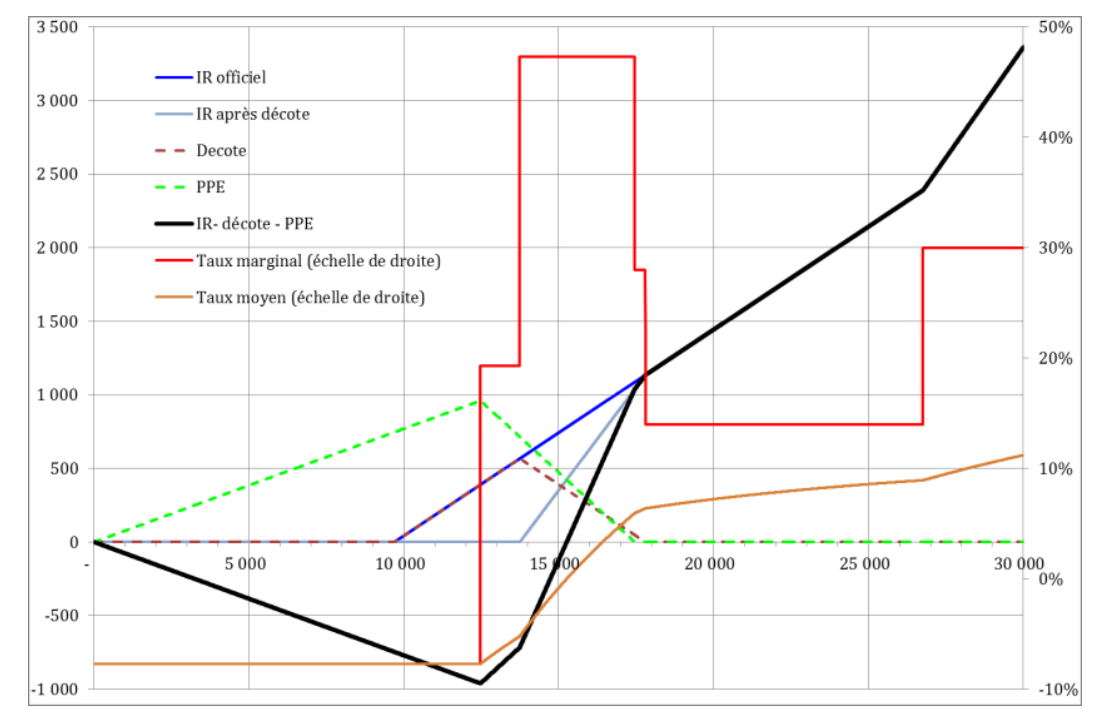

Figure 9 : Barème sur les revenus 2015 pour un célibataire sans enfant et sans allocation logement de l'impôt sur le revenu, de la décote et de la prime pour l'emploi. Calculs de l'auteur.

Malgré l'extrême simplicité de ce cas type, on constate que la combinaison de ces trois dispositifs suffit à produire une courbe de taux marginal erratique. Celui-ci est négatif à $-7,7 \%$ jusqu'à ce que l'on atteigne le sommet de la Prime pour l'emploi à $12475 €$, qui correspond au revenu imposable d'un salarié à temps plein rémunéré toute l'année au SMIC. On entre alors dans la phase de sortie de la PPE au taux 19,3\%, puis dans la sortie à partir de $13744 €$ de la décote au taux de 28\%. La combinaison des deux entraine alors un taux marginal de 47,3\% jusqu'à la sortie de la PPE et à la fin de la décote à respectivement $17451 €$ et $17797 €$. Seul compte alors le barème officiel de l'impôt sur le revenu avec un taux marginal de $14 \%$ jusqu'à $26764 €$ puis à $30 \%$ jusque $71754 €$.

Les exercices de fiscalité optimale décomposent souvent les déterminants des taux marginaux en un facteur d'efficacité qui dépend des réponses intensives, un facteur redistributif qui dépend de la moyenne des poids sociaux des effets revenus et des réponses extensives et un terme de distribution qui correspond au coefficient de Pareto de la distribution des revenus (Diamond (1998), Saez (2001,2002)). Chacun de ces termes évoluent le long de la distribution des revenus, permettant alors de mieux comprendre pourquoi un barème non-linéaire d'imposition des revenus s'avère nécessaire. On peut alors rationnaliser des barèmes avec des taux marginaux négatifs en bas, notamment si les réponses extensives de l'offre de travail prédomine et que l'on souhaite redistribuer sur les pauvres qui travaillent (Saez (2002)). On peut également rationnaliser des barèmes avec des taux marginaux en bas extrêmement élevés (Saez (2001)) et un profil en U des taux marginaux en optimaux (Diamond (1998)). Ce sera par exemple le cas si l'on veut des transferts qui soient à la fois importants et concentrés sur les bas salaires qui nécessitent alors d'accepter que les transferts diminuent rapidement avec les revenus, faisant ainsi apparaître des taux marginaux élevés. En revanche, il semble difficile de rationaliser que l'évolution des trois termes d'efficacité, redistributif et de distribution puissent justifier à la fois un taux marginal négatif jusqu'à $12475 €$ qui monte ensuite pour atteindre

${ }^{12}$ Ce graphique n'est qu'illustratif. Il néglige en particulier le rôle des cotisations sociales et de leurs allègements ainsi que de la CSG. 
$47 \%$ entre $13743 €$ et $17451 €$ pour redescendre ensuite à $14 \%$ entre $17797 €$ et $26764 €$ et remonter ensuite à $30 \%$. On le voit, le profil auquel font actuellement face les célibataires sans enfant ne bénéficiant pas des allocations logement et ne recourant pas au RSA n'est vraisemblablement pas déterminé par des considérations liées à la recherche optimale. Il semble bien au contraire que chacune de ses composantes ait été pensée indépendamment des autres. Le manque de réflexion sur l'interaction des différents dispositifs crée alors un flou rendant opaque les incitations à la reprise d'activité, et risquant fort de ce fait de nuire à leur efficacité.

\section{Vers une réforme d'ensemble}

La première partie a montré à partir de l'expérience de l'EITC aux États-Unis que la politique d'incitation au retour d'activité pour les bas salaires a besoin de transparence pour être comprise. La description des différents dispositifs existant en France concernant les bas salaires a montré au contraire la présence d'un millefeuille qui obscurcit grandement le message incitatif. L'idée de fusionner en un seul barème l'ensemble de ces dispositifs semble donc s'imposer. Pourtant, un certain nombre de difficultés se présente face à une telle simplification. En examinant chacune de ces difficultés, nous allons petit à petit formuler une proposition susceptible à la fois de clarifier les incitations à la reprise d'activité tout en étant réalisable.

En France, ce sont les salaires bruts qui apparaissent dans les contrats de travail et dans les conventions collectives. C'est également le SMIC brut qui est légalement déterminé. Aussi, tout transfert de charges entre les cotisations sociales employeurs vers l'ensemble constitué par les cotisations sociales salariés, la CRDS, la CSG, l'impôt sur le revenu, net de l'ensemble PPE-RSA activité aura des implications sur le coût du travail et le salaire super-net, du moins à court terme. C'est pourquoi il nous semble préférable de proposer la création de deux barèmes uniques portant sur l'intégralité des revenus d'activité, à savoir :

- Un barème «employeur unique » qui reprendrait l'actuel barème de cotisations sociales employeurs auquel viendrait se soustraire les allègements « Fillon » sur les bas salaires, les réductions de charges héritées du Crédit d'Impôt pour la Compétitivité et l'Emploi et celles associées au pacte de responsabilité.

- Un barème "employé unique » qui reprendrait l'actuel barème de cotisations sociales employés, la CSG, la CRDS, l'impôt sur le revenu, auquel viendrait se soustraire la prime d'activité.

Un tel système serait très facile à mettre en œuvre si tous les contribuables étaient des célibataires sans enfants, ne recevant que des revenus d'activité. Le montant de ces deux barèmes ne dépendrait alors que du revenu d'activité brut que les employeurs doivent dès à présent déclarer mensuellement pour le paiement des cotisations sociales. Le cas des travailleurs indépendants et des professions libérales ne poserait pas davantage de difficultés que le prélèvement actuel de leurs cotisations sociales. Le système proposé présenterait ainsi l'énorme avantage d'opérer des prélèvements à la source et qui seraient contemporains de la réalisation des revenus. En particulier, le bénéfice de la Prime d'Activité serait perçu automatiquement et sans procédure administrative ni stigmatisation. On résoudrait ainsi le problème de la PPE qui était versée avec retard et du RSA activité avec son danger de stigmatisation et son fort taux de non-recours. Du côté des employeurs, le bénéfice des allègements du CICE ou du pacte de compétitivité serait contemporain et automatique, ce qui rendrait également les incitations plus transparentes (cf. Lehmann et L'Horty (2014)).

Cette proposition de deux barèmes présente le défaut de faire disparaitre le lien entre le paiement des cotisations sociales et les prestations sociales que financent les cotisations sociales. Aussi, dans le but de maintenir cette philosophie Bismarckienne de la protection 
sociale, on pourrait exclure de cette fusion dans les deux barèmes susmentionnés les cotisations sociales dites «contributives » qui sont les seules cotisations sociales associées réellement à un prestation sociale pour le cotisant. C'est notamment le cas des cotisations de la branche vieillesse qui financent directement un revenu différé sous la forme de pension de retraite. On peut admettre que la même logique existe pour la partie des cotisations de la branche santé servant à indemniser les absences pour maladie, par opposition au remboursement de soin qui sont devenus quasiment universels, notamment par le biais de la CMU. Les cotisations à l'assurance chômage peuvent également être qualifiées de contributives, encore que le risque de chômage étant surtout concentré sur les populations les moins diplômées et les plus jeunes, la branche chômage comprend également une dimension redistributive non négligeable, justifiant d'ailleurs la prise par l'Etat du régime de solidarité chômage. En revanche, les prestations que financent la branche «famille» de la sécurité sociale ne sont en rien liées aux cotisations précédemment versées par les bénéficiaires. Ainsi, même si la frontière entre cotisations sociales contributives et non contributives reste à clarifier, on peut exclure les cotisations sociales contributives du périmètre des deux barèmes uniques susmentionnés et restreindre le périmètre de la fusion en deux barèmes aux seules cotisations sociales non contributives.

Une autre difficulté vient de la crainte qu'une éventuelle fusion fragiliserait le financement de la protection sociale. En effet, la base de l'impôt sur le revenu étant mitée par des niches fiscales beaucoup plus nombreuses et coûteuses que celles existant pour les cotisations sociales et la CSG (Landais et al. (2011), CPO (2015)), la peur que le « mauvais impôt ne chasse le bon » constitue un obstacle récurrent à tout projet de fusion de l'IR avec des prélèvements finançant la sécurité sociale. Il existe pourtant des techniques juridiques pour garantir aux organismes de sécurité sociale qu'une fraction des prélèvements fusionnés leur soit affectée. Le Conseil des prélèvements obligatoires (CPO (2015) page 114) indique qu'il serait en particulier possible de recourir à une « affectation a priori d'une fraction d'un nouvel impôt à la sécurité sociale » à l'instar de ce qui se pratique déjà notamment avec la CSG, la TVA ou les taxes sur le tabac.

La présence de sources de revenus multiples constitue indubitablement une autre difficulté que doit d'ailleurs affronter tout prélèvement obligatoire non proportionnel prélevé à la source. En cas d'employeurs multiples, les prélèvements «employés-unique» et "employeurs-unique» opérés auprès d'un employeur devraient alors dépendre des autres revenus d'activité. Deux réponses sont possibles. La première consiste à ce que chaque entreprise opère des prélèvements sur la base des seuls revenus bruts qu'elle verse et que l'administration fiscale procède périodiquement à un rattrapage, sur une base annuelle ou trimestrielle. Une deuxième réponse consisterait à ce que l'administration fiscale communique à chaque employeur un taux moyen synthétique de prélèvement, selon la procédure actuellement envisagée pour 2017 à propos du passage au prélèvement à la source de l'impôt sur le revenu.

La présence de revenus du capital pose une difficulté spécifique car on pourrait vouloir appliquer un barème différent aux revenus du capital et aux revenus du travail. Une telle option peut en particulier être retenue si l'on souhaite mettre en place une réforme fiscale qui n'alourdisse pas la fiscalité du capital. Comme notre but dans cet article est de ne proposer qu'une réforme de tuyauterie sur l'architecture des dispositifs et non une réforme de tuyaux portant sur les taux de taxation, il nous semble important de proposer une architecture de prélèvements obligatoires qui autorisent ensuite soit à augmenter soit à diminuer la fiscalité sur les revenus du capital. Il faudrait alors mettre en place un troisième barème qui serait spécifique à ces derniers. Rappelons qu'à l'heure actuelle, la CSG portant sur les revenus du patrimoine et les plus-values est déjà un taux de $8.2 \%$ supérieur au taux de $7.5 \%$ portant sur les revenus d'activité.

La difficulté soulevée par les dépenses fiscales existant actuellement dans le barème de l'impôt sur le revenu peut également être contournée. Si Landais et al. (2014) faisaient de la 
remise à plat des niches fiscales un argument fort en faveur de la fusion de l'IR et de la CSG qu'ils appelaient de leurs vœux, nous préférons proposer une réforme qui ne ferme pas la porte au maintien des dépenses fiscales que l'on jugerait les plus utiles. Notre point de vue est que la fusion est nécessaire pour une question de transparence des incitations fiscales, indépendamment de la question de la remise en cause des niches fiscales. Notre proposition consisterait à sortir les niches fiscales des barèmes spécifiques aux revenus d'activité d'imposition pour en faire des instruments de politiques économiques autonomes et déconnectés de la redistribution des revenus d'activité. Notre point de vue et que si l'Etat souhaite par exemple subventionner les travaux de rénovation pour économie d'énergie, l'investissement dans les DOM-TOM ou encourager l'accession à la propriété, qu'il le fasse par des transferts spécifiques dont les montants dépendent des dépenses effectuées, mais ne dépendent pas du revenu des agents. Ce sera là une bonne application du principe « un objectif, un instrument de politique économique ».

La principale difficulté concerne donc le traitement de la famille. Cette question comprend d'abord le traitement des enfants. Comme nous l'avons déjà vu, la combinaison des différentes politiques aboutit à une situation qui n'est plus très éloignée de celle dans laquelle les parents recevraient une aide forfaitaire dépendant du nombre d'enfants et ne dépendant plus de leurs revenus (cf. Figure 8 tiré de Boisnault et Fichen). L'idée serait ainsi de faire sortir les aides aux familles des transferts des revenus. $\mathrm{Au}$ lieu d'attribuer des demi-parts supplémentaires par personne à charge ou de faire dépendre le barème de la prime d'activité du nombre d'enfants, mieux vaut aller au bout de la logique du plafonnement du quotient familial en faisant reposer l'aides aux familles sur les seules allocations familiales qui seraient alors non imposables.

La difficulté serait alors surtout la question du quotient conjugal : doit-on converger vers un barème individuel, à l'instar de celui pour lequel milite Landais et al. (2011) ou de conserver la tradition française d'un barème au niveau du foyer fiscal ? Du point de vue des incitations, l'argument habituel est qu'il vaut mieux privilégier un barème individuel afin que le second apporteur de revenus, souvent les épouses, puisse bénéficier de taux marginaux plus faibles. Comme les épouses ont tendance à se caractériser par des élasticités plus élevées de l'offre de travail, ce serait un moyen d'améliorer les incitations à la reprise d'emploi. Notons toutefois qu'un tel argument est valable si et seulement si le barème d'imposition est convexe si bien que les taux marginaux augmentent avec les revenus. C'est évidemment le cas avec le seul impôt sur le revenu, mais nous avons vu que la sortie de la prime pour l'emploi rend les taux marginaux décroissant entre 1 et 1.4 SMIC. Un autre argument repose sur le fait que les décisions d'offre de travail sont surtout concentrées le long de la marge extensive, auquel cas ce qui compte n'est pas le taux marginal d'imposition mais le taux de taxation de l'emploi, i.e. la somme du taux de taxation que paye un salarié et de la prestation sociale qu'il recevrait si il était sans emploi sur le salaire. Si ce taux de taxation de l'emploi augmente avec le revenu, alors le second apporteur de revenu aurait davantage intérêt à travailler si le barème était individualisé en lieu et place d'un impôt sur le revenu au niveau du foyer fiscal. Les estimations micro-économétriques de Carbonnier qui exploitent le quotient conjugal de l'impôt sur le revenu français pour estimer l'élasticité extensive de l'offre de travail du second apporteur de revenu invitent à ne pas sousestimer les bienfaits d'un barème individualisé en termes d'emploi.

On objectera néanmoins qu'une autre logique plaiderait plutôt pour calculer au niveau du ménage l'imposition des revenus du capital. En effet, avec un barème individuel, il conviendrait de fixer une règle pour déterminer à quel époux attribuer les revenus issus des actifs que possède le ménage. Le risque est en effet important que si on laisse trop de liberté aux foyers pour trancher cette question, ceux-ci attribuent les revenus du capital à l'époux ayant les revenus d'activité les plus faibles afin de réduire le taux auquel serait imposé les revenus du capital. La réponse serait ou bien d'imposer strictement la règle obligeant chaque époux d'un ménage à déclarer la moitié des revenus du patrimoine dont bénéficie le couple, soit d'appliquer 
au niveau du foyer fiscal et non des individus le barème sur les revenus du patrimoine. On aurait ainsi une imposition des revenus d'activité ou niveau des individus, et une imposition des revenus du capital au niveau des ménages. Néanmoins, ce risque disparaitrait si les revenus du capital était imposés selon un barème spécifique, indépendamment des revenus d'activité, à l'instar de ce qui se pratique par exemple au Danemark.

Le principal obstacle qui reste à discuter concerne la constitutionnalité de notre proposition de fusion en trois barèmes. La jurisprudence du Conseil constitutionnel semble s'attacher à vérifier que l'égalité de tous devant l'impôt soit appréciée au niveau du foyer fiscal et non au niveau des individus (CPO 2015). Cette jurisprudence est notamment à l'origine de la complexité de la $\mathrm{PPE}$, avec un rattachement à l'IR mettant à mal son versement contemporain là où le projet initial de ristourne dégressive de CSG aurait garanti une subvention versée de manière contemporaine et automatique. On peut être quelque peu surpris par le poids de la contrainte que fait peser le Conseil constitutionnel sur les possibilités de réformes fiscales. La jurisprudence du Conseil constitutionnel en matière d'imposition repose en effet essentiellement sur son interprétation de l'article 13 de la déclaration des droits de l'homme et du citoyen de 1789 qui prévoit l'égalité de tous devant l'impôt. Cela signifie que deux unités imposables ayant les mêmes facultés contributives doivent payer le même impôt. En 1789, les seules taxes qui existaient étaient proportionnelles et il n'était question ni de progressivité de l'impôt, ni d'incitation à la reprise d'activité. Savoir si les capacités contributives devaient être évaluées au niveau individuel ou familial n'était certainement pas la préoccupation première de l'époque. Pourtant, au fil du temps, le Conseil constitutionnel a estimé que c'est au niveau du foyer fiscal et non au niveau des individus que le principe d'égalité de tous devant l'impôt devait s'appliquer. On peut donc s'inquiéter, à l'instar de Collet (2014), de l'étendu du pouvoir que ce sont progressivement octroyés les « sages » en matière fiscale. Pourtant, l'interprétation de cette jurisprudence est-elle-même discutable. En effet, le Conseil constitutionnel ne s'est pas opposé à ce que la CSG applique des taux différents à différents types de revenus, créant ainsi une forme de redistribution entre les ménages selon le type de revenus qu'ils touchent. La CSG crée donc une inégalité de traitement entre des foyers ayant le même revenu total si la source de ces revenus est différente. Par ailleurs, certains auteurs comme Collet et Racine (2015) estiment qu'une individualisation de l'imposition des revenus des personnes ne serait pas forcément censurée par le Conseil constitutionnel si le législateur assumait explicitement cette individualisation.

Face à ce risque constitutionnel qui semble constituer la principale difficulté à la mise en place d'une simplification, nous proposons finalement non pas une mais deux réformes alternatives.

La première réforme consisterait à remplacer l'IR, la PA, la CSG, la CRDS, les cotisations sociales non contributives employeurs et employés, le CICE, le pacte de responsabilité et les allègements bas salaires Fillon en trois prélèvements à la source selon des barèmes non proportionnels :

- Un prélèvement « employeurs sur les revenus d'activité » prélevé à la source et de manière contemporaine sur une base individuelle qui viendrait se substituer aux différentes cotisations patronales non contributives aux réductions de charges sur les bas salaires dites Fillon, ainsi que le CICE et le pacte de responsabilité.

- Un prélèvement "employé sur les revenus d'activité » prélevé à la source et de manière contemporaine sur une base individuelle, qui viendrait se substituer à l'actuelle imposition des revenus d'activité dans l'IR, à la CSG et la CRDS sur les revenus d'activité, aux cotisations sociales salariées non contributives et à la Prime d'Activité. 
A la recherche des incitations perdues...

- Un prélèvement « revenus du capital » qui viendrait se substituer à l'actuelle imposition des revenus du capital dans l'IR, à la CSG, à la CRDS et aux différents prélèvements sociaux portant sur les revenus du capital.

Le versement d'allocations familiales ainsi qu'un certain nombre de dépenses fiscales incitatives se ferait indépendamment de l'imposition des revenus. Une telle proposition réformerait explicitement le caractère familial de l'imposition des revenus d'activité.

Cette proposition fait l'hypothèse que le Conseil constitutionnel se satisferait $\mathrm{du}$ caractère explicite de l'individualisation de l'imposition des revenus d'activité que cette réforme assumerait.

On ressent néanmoins le besoin de formuler une proposition alternative, aboutissant à une architecture moins transparente, mais soulevant moins de difficultés sur le plan constitutionnel. Dans cette deuxième proposition, on conserverait à l'identique la proposition d'un barème unique "employeurs sur les revenus d'activité ». En effet, la jurisprudence constitutionnelle a toujours considérer les cotisations patronales comme étant à la charge des entreprises et ne s'est donc jamais ému que les cotisations sociales patronales sont devenues non-proportionnelles sans tenir compte des autres revenus En revanche, notre proposition de barème " employé sur les revenus d'activité » ne serait maintenue que comme acompte d'un impôt sur le revenu qui serait maintenu au niveau du foyer fiscal et dont les montants seraient calculés à la fin de chaque année une fois connu l'intégralité des revenus des ménages. Cette proposition fait écho à la proposition formulée notamment par Alain Trannoy (2012) de transformer la CSG en acompte de l'impôt sur le revenu. Elle repose sur le fait que les systèmes de prélèvements à la source de l'impôt sur le revenu qui existent de par le monde prévoient très fréquemment des procédures de régularisations annuelles le temps pour l'administration fiscale de parfaitement connaître l'intégralité de la situation des assujettis. On aurait alors des prélèvements sur les revenus d'activité qui seraient opérés mensuellement sur une base individuelle et qui seraient complétés par un correctif de fin d'année. Le montant de ce correctif serait égal à la différence entre l'impôt total dû au niveau du foyer sur la base de l'ensemble des revenus du foyer et le montant total des prélèvements déjà perçus à la source. L'imposition du capital serait alors opérée au moment de ce correctif de fin d'année. Le risque constitutionnel nous semble pouvoir être écarté avec cette proposition puisque le montant final de l'impôt est calculé au niveau du foyer fiscal en fonction de l'ensemble des revenus du foyer. On conserverait néanmoins le bénéfice d'une prime d'activité qui serait versée de manière automatique et contemporaine.

\section{Bibliographie}

Tony Atkinson et Joseph Stiglitz [1976] : The Design of Tax Structure: Direct Versus Indirect Taxation, Journal of Public Economics, 6 (1-2), 55-75.

Olivier Bargain [2014] : Les enjeux budgétaires et économiques de la réforme de l'imposition des revenus des ménages, partie 2 : enjeux et principes d'une refonte du système redistributif français, rapport particulier pour le conseil des prélèvements obligatoires.

Robin Boadway et Jean-François Tremblay [2013] : Optimal Income Taxation and the Labour Market: An Overview, CESifo Economic Studies, 59 (1), 93-148.

Denis Boisnault et Anne Fichen [2015] : Enfants, politique familiale et fiscalité : les transferts du système socio-fiscal aux familles en 2014, Trésor-Eco 142. 
A la recherche des incitations perdues...

Clément Carbonnier [2014] : The influence of taxes on employment of married women, evidence from the French joint income tax system, LIEPP working paper $\mathrm{n}^{\circ} 23$.

Raj Chetty [2015] : Behavioral Economics and Public Policy: A Pragmatic Perspective, American Economic Review, Ely lecture Papers \& Proceedings, 105 (5), 1-33.

Martin Collet [2014] : L'impôt confisqué, Odile Jacob.

Martin Collet et Pierre-François Racine [2015] : Imposons les individus, pas les foyers, Le Monde économie 5 février 2015.

Gabrielle Fack [2006] : Are Housing Benefits an Effective Way to Redistribute Income? Evidence From a Natural Experiment in France, Labour Economics 13(6), 746-771.

Henri Guillaume [2011], Rapport du Comité d'évaluation des dépenses fiscales et des niches sociales présidé par Henri Guillaume.

Conseil des Prélèvements Obligatoires (CPO) [2015]: Impôt sur le revenu, CSG: Quelles réformes?

Peter Diamond [1980] : Income Taxation with Fixed Hours of Work, Journal of Public Economics, 13 (1), 101-110.

Emmanuel Fahri et Xavier Gabaix [2015] : Optimal Taxation with Behavioral agents, NBER working paper 21524 .

Hilary W. Hoynes et Ankur J. Patel [2015] : Effective Policy for Reducing Inequality? The Earned Income Tax Credit and the Distribution of Income, NBER working paper 21340.

Camille Landais, Thomas Piketty et Emmanuel Saez [2011] : Pour une révolution fiscale, un impôt sur le revenu pour le XXIème siècle, Le seuil / République des idées.

Etienne Lehmann, Yannick L'Horty [2014] : Renforcer la progressivité des prélèvements sociaux, Revue Française d'Economie, 24, 25-61.

Etienne Lehmann, Laurent Simula et Alain Trannoy [2014] : Tax Me if You Can! Optimal Nonlinear Income Tax between Competing Governments, Quarterly Journal of Economics, 129 (4), 1995-2030.

Thomas Piketty [1997]: La redistribution fiscale face au chômage, Revue Française d'Economie, 12 (1), 157-201.

James Mirrlees [1971] : An Exploration in the Theory of Optimum Income Taxation, Review of Economic Studies, 38 (114), 175-208.

Emmanuel Saez [2002] : Optimal Income Tax transfer Programs: Intensive versus Extensive Labor Supply Responses, Quarterly Journal of Economics, 117 (3), 1039-1073.

Emmanuel Saez [2010] : Do Taxpayers Bunch at Kink Points?, American Economic Journal: Economic Policy, 2(3), 180-212.

Alain Trannoy [2012] : Il faut une révolution fiscale, qu'en pensent les économistes ? Collection on entend dire que, Eyrolle, Les Echos. 
A la recherche des incitations perdues...

Alain Trannoy [2015] : La décote détricote l'impôt sur le revenu, tribune publiée le 14 septembre 2015 sur Lemonde.fr 
15-4. Crise économique, durée du chômage et accès local à l'emploi : Eléments d'analyse et pistes d'actions de politique publique locale

Mathieu Bunel, Elisabeth Tovar

15-3. L'adresse contribue-t-elle à expliquer les écarts de salaires ? Le cas de jeunes sortant du système scolaire

Emilia Ene Jones, Florent Sari

15-2. Analyse spatiale de l'espace urbain : le cas de l'agglomération lyonnaise Emilie Arnoult, Florent Sari

15-1. Les effets de la crise sur les disparités locales de sorties du chômage : une première exploration en Rhône-Alpes

Yannick L'Horty, Emmanuel Duguet, Florent Sari 
14-6. Dépréciation du capital humain et formation continue au cours du cycle de vie : quelle dynamique des externalités sociales?

Arnaud Chéron, Anthony Terriau

14-5. La persistance du chômage ultra-marin

Yannick L'Horty

14-4. Grèves et productivité du travail : Application au cas français Jérémy Tanguy

14-3. Le non-recours au RSA "socle seul": L'hypothèse du patrimoine Sylvain Chareyron

14-2. Une évaluation de l'impact de l'aménagement des conditions de travail sur la reprise du travail après un cancer

Emmanuel Duguet, Christine Le Clainche

14-1. Renforcer la progressivité des prélèvements sociaux

Yannick L'Horty, Etienne Lehmann 
La Fédération de recherche CNRS Travail, Emploi et Politiques Publiques (TEPP, FR $\mathbf{n}^{\circ} \mathbf{3 4 3 5}$ ) réunit des centres de recherche en économie et sociologie :

- Le Centre d'Etudes des Politiques Economiques de l'université d'Evry, EPEE, Université d'Evry Val d'Essonne

- Le Centre Pierre Naville, CPN, Université d'Evry Val d'Essonne

- Le Centre de Recherche en Economie et Management, CREM, Université de Caen Basse Normandie et Université de Rennes 1

- L'Equipe de Recherche sur les Marchés, l'Emploi et la Simulation, ERMES, Université deParis II Panthéon-Assas

- L'Equipe de Recherche sur l'Utilisation des Données Temporelles en Economie, ERUDITE, Université de Paris-Est Créteil et Université de Paris-Est Marne-la-Vallée

- Le Groupe d'Analyse des Itinéraires et des Niveaux Salariaux, GAINS, Université du Maine

La Fédération TEPP rassemble 150 chercheurs et enseignants-chercheurs, 140 doctorants et 40 chercheurs associés, qui étudient les mutations du travail et de l'emploi en relation avec les choix des entreprises et analysent les politiques publiques en mobilisant les nouvelles méthodes d'évaluation. 